\title{
Relation between flow-pressure-diameter studies in experimental stenosis of rabbit and human small bowel
}

\author{
P Morel, J Alexander-Williams, A Rohner
}

\begin{abstract}
Patients with inflammatory and ischaemic bowel diseases seem to tolerate narrowing of the gut lumen to a critical degree of stenosis without obstructive symptoms. To determine the physical factors involved in bowel occlusion, we created an experimental model using New Zealand rabbits in acute experiments under general anaesthesia. At operation a loop of small bowel was isolated and canulated, proximally for perfusion and pressure recording and distally to monitor flow. Having established the physiological pressure and flow conditions in a normal loop of gut, a stenosis was created using circular adjustable rings of determined widths. Pressure and flow were measured constantly and the variables studied were luminal diameter, stenosis length, and perfusate viscosity. This experimental model was reproduced using resected segments of human small bowel. We found a critical point at $60 \%$ of the original diameter - down to which the small bowel is able to maintain normal flow. At a diameter smaller than this, the physiological parameters are rapidly altered up to the point of complete obstruction. In the rabbit model bowel rupture occurs at $30 \%$ of the initial size. Increased viscosity of the fluid and length of the stenosis alter this critical point inducing a larger critical diameter. We did not observe any cumulative effect of multiple identical stenoses.
\end{abstract}

In humans the healing of ulcerative diseases of the gut often leads to fibrotic stenosis. Examples of these diseases are chronic peptic ulceration of the oesophagus, pylorus, and duodenum; chronic inflammatory bowel diseases such as tuberculosis and Crohn's disease; and healing acute ulcerations such as potassium and ischaemic ulcerations. Although the resulting stenoses occur progressively, at first they are usually clinically undetectable. There must therefore be a critical degree of narrowing or a critical luminal diameter at which stenosis begins to cause symptoms of obstruction. If it were possible to determine and measure the critical diameter, we might anticipate or even avoid symptoms by early operation or dilatation in patients developing stenosis.

The aim of the study was to assess the critical diameter at which pressure rises sufficiently to be potentially damaging to bowel mucosa.

The factors that affect the passage of fluid through the gut include pressure, internal diameter, content viscosity, and the friction coefficient of the mucosa. Although some of these measurements can be made in vitro in the laboratory, it is more appropriate to study these variables on living tissues.

\section{Materials and methods}

Thirty six New Zealand rabbits with a body weight of 3 to $3.5 \mathrm{~kg}$ were anaesthetised with fentanyl-fluanisone (Hypnorm) $0.3 \mathrm{ml} / \mathrm{kg}$ intramuscularly. This allowed spontaneous breathing.

Before performing the stenosis experiments, we studied normal bowel movements of the gut in 12 rabbits by measuring pressure and flow. The variation of pressure and its mean measured value were recorded after closure of the abdomen by a pressure transducer introduced at laparotomy into a jejunal loop. Thereafter we reopened the abdomen, transected the proximal jejunum, and connected a pump to perfuse the proximal jejunum with a normal saline solution. The speed of the pump was adjusted to produce the above mentioned mean pressure in the jejunum. At this pressure the established pump output allowed us to determine the jejunal flow. We then reproduced experimental conditions by perfusing the bowel with the same device.

In the stenosis experiments, a $15 \mathrm{~cm}$ long jejunal loop was isolated; its ends were transected but the blood supply was preserved. Both ends were canulated. An Ismatec pump was used to perfuse the jejunum with fluids of various viscosities, beginning with normal saline and progressing to higher viscosity solutions. Variable sized stenoses were produced in the jejunal loop using circular external adjustable rings. The rings were progressively narrowed using a screw system connected to an electrical resistance. Each diameter of the ring correlated with a different value of resistance measured with an ohmmeter. This electrical device was calibrated using metallic tubes of known diameter inserted within the gut using 12 different jejunal loops in four animals. Distal to the stricture, a collecting tube connected to a graduated collector allowed the measurement of flow passing the stenosis. The pressure transducer was introduced proximal to the stenosis and connected to a recorder (Siemens Mingograph 803) (Fig 1).

The modified variables were the diameter of the stenosis, its length, and the viscosity of the fluid. We recorded pressure proximal to the stenosis, and flow measurements were performed at multiple points on the scale for each variable in turn. Using this technique, the recordings from 36 jejunal loops were analysed under standard conditions. 


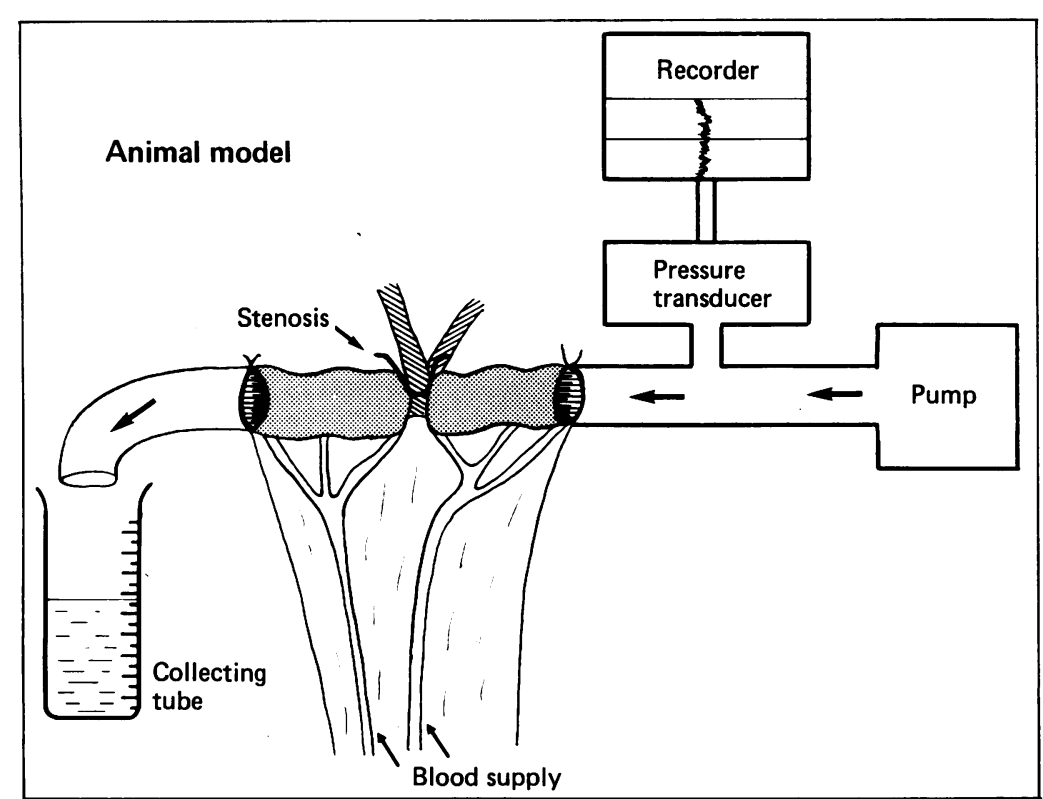

Figure 1: The rabbit animal model.
This experiment was approved by the official University Hospital Geneva Animal Welfare Committee.

We performed a preliminary study using the same method on 12 small bowel segments immediately after their resection to assess the correlation between our animal model and the human gut. Seven segments were of terminal ileum resected at the time of a right hemicolectomy performed for tumours (5) or ulcerative colitis (2); five were macroscopically healthy looking areas of gut located between stenoses in patients with Crohn's disease. The loops were 5 to $8 \mathrm{~cm}$ long and in them we assessed a $1 \mathrm{~cm}$ long stenosis perfused with normal saline. We used the same flow rate as in the animal experiments as we were not aware of reliable data on the flow rates in the human gut. In patients with a watery ileostomy effluent but without an obstructed bowel we observed rates of $50 \mathrm{ml} /$ minute.

\section{Results}

\section{ANIMAL EXPERIMENTS}

Determining basal conditions. After closure of the abdomen, the physiological pressures recorded with the catheter introduced in a jejunal loop ranged from 2.4 to $9.5 \mathrm{~cm} \mathrm{H}_{2} \mathrm{O}$, with a mean measured pressure of $3.4 \mathrm{~cm} \mathrm{H}_{2} \mathrm{O}$. The highest pressures occurred during bowel contraction.

Under mean pressure, the flow of a normal saline solution was $42 \mathrm{ml} /$ minute.

These conditions of pressure and flow were considered the basal state for our subsequent experiments.

Relation between the diameter of the stenosis and the proximal pressure. The loop was perfused at a constant flow of $41 \mathrm{ml} /$ minute, and the diameter of the stenosis was adjusted while recording proximal intraluminal pressure.

We observed that the diameter of the stenosis could be reduced to $60 \%$ of its original size without any appreciable change in the pressure. From this diameter downwards, pressure increased exponentially with the reduction in the lumen size down to a rupture point at a diameter

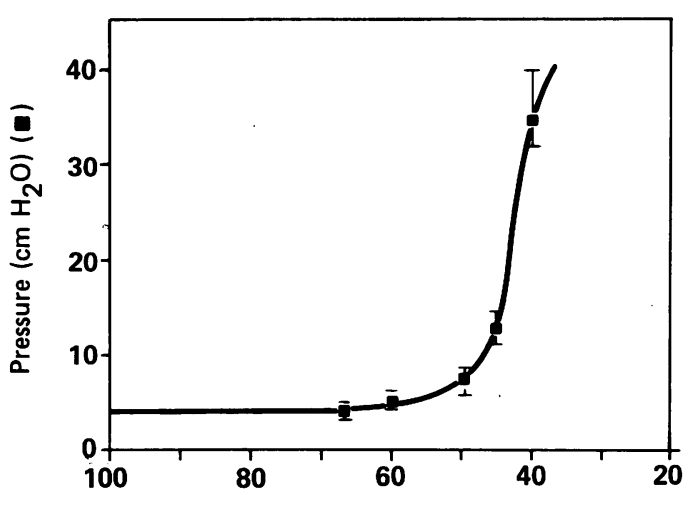

Luminal diameter (\% of normal diameter)

Figure 2: Relation between luminal diameter and pressure during normal saline perfusion of rabbit jejunum. $\mathbf{Q}=$ mean; $\mathrm{I}=$ standard deviation.

of $30 \%$ of the original (Fig 2). The rupture was usually a longitudinal split of some millimetres long just proximal to the stenosis.

Relation between the diameter of the stenosis and flow. The gut was perfused under a constant pressure of 3 to $4 \mathrm{~cm} \mathrm{H}_{2} \mathrm{O}$ and flow was recorded at the variable diameters of stenosis.

We obtained a constant and normal flow down to nearly the same critical diameter recorded previously in the pressure measurement. From this point, the flow decreased rapidly down to a complete obstruction at the level of $35 \%$ of the normal diameter (Fig 3).

Changing the viscosity of the perfused solution.

The normal saline solution used was replaced by progressively higher viscosity solutions by thickening with glycerine. The viscosity of the fluid was determined in seconds-din. The viscosity measurement is the time in seconds taken for the contents of a $100 \mathrm{ml}$ container to empty through a $1 \mathrm{~mm}$ hole. Under the same experimental conditions we observed a slightly higher basal pressure and the flow and pressure curves shifted towards the left with the same critical point changes (Fig 4).

Changing the length and number of stenoses. We studied three lengths of stenoses: $4 \mathrm{~mm}, 8 \mathrm{~mm}$, and $12 \mathrm{~mm}$, and observed that increasing length correlated with a larger critical diameter (Fig 5).

Creating several stenoses on the same loop, all with the same diameter and length, did not alter the 'critical point', which remained that of the

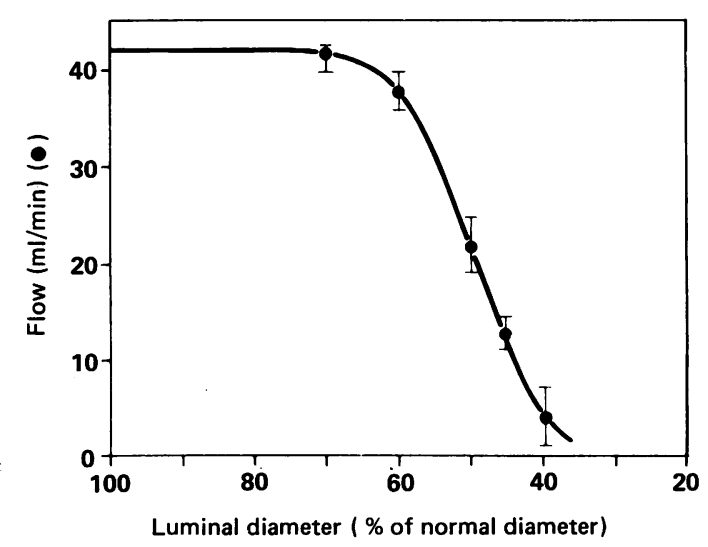

Figure 3: Relation between luminal diameter and flow during normal saline perfusion of rabbit jejunum. $=$ mean; $\mathrm{I}=$ standard deviation. 
Figure 4: Effects of variation of the viscosity of the perfused intestinal fluid on pressure and flow. Changes produced by high viscosity of the consistency of thick soup $(19 \cdot 5$ sec.-din $)$. Mean values only shown.

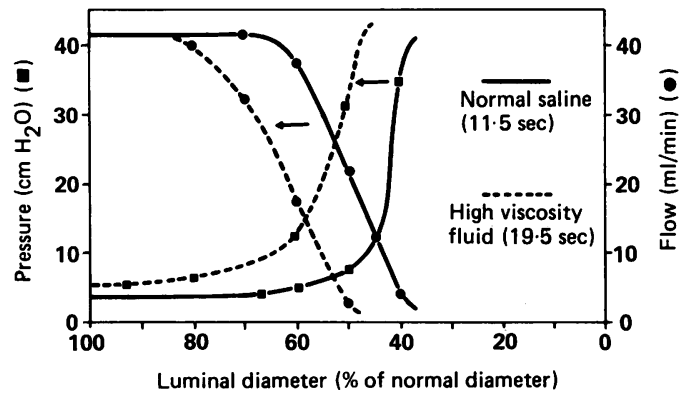

proximal stricture. In other words, there was no cumulative effect of several identical stenosis.

HUMAN SMALL BOWEL (PRELIMINARY RESULTS) The 12 segments studied gave statistically similar results. They suggested that for a $1 \mathrm{~cm}$ length stricture perfused with normal saline, 15 $\mathrm{mm}$, or $60 \%$ of the initial diameter, is the critical diameter at which proximal pressure rises with a decreased flow (Fig 6).

\section{Discussion}

In the animal experiment we showed a critical diameter down to which rabbit small bowel is able to maintain conditions suitable for normal intestinal flow. At a diameter smaller than this, physiological parameters are rapidly and dramatically altered up to the point of complete obstruction. Factors which change this critical luminal size are viscosity of the fluid and length of the stenosis. The number of stenoses does not change this critical diameter.

In our experiment we used an acute mechanical obstruction of the gut. This differs from physiological in vivo obstruction because we perfused the stenosis with a pump instead of using normal propulsive bowel movements. The reactions of the gut to obstruction have been described. ${ }^{1-3}$ We know that motor activity in obstructed bowel is characterised by successive phases. In the early phase of partial obstruction there is an increase in myoelectrical spike bursts proximal to the stenosis and a simultaneous decrease distally. At this stage the flow passing the stenosis is already below normal and the proximal pressure is increased. In the subsequent phase, beginning four to six hours later, prolonged periods of absent myoelectrical spike activity are interrupted by clusters of intense spike activities. ${ }^{\prime}$ At this stage, the proximal bowel propulsive force is reduced and there is

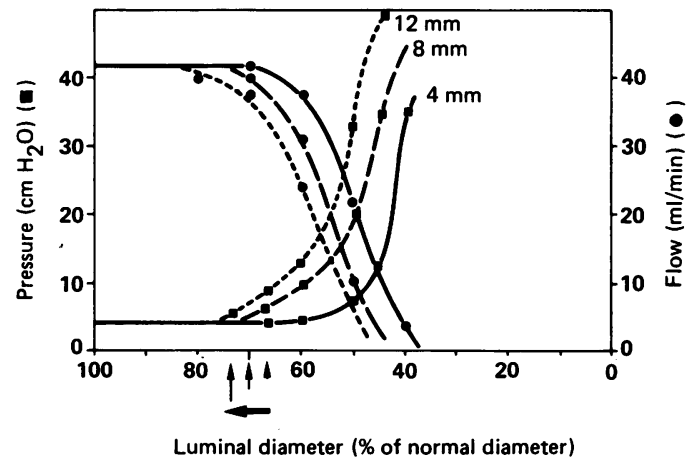

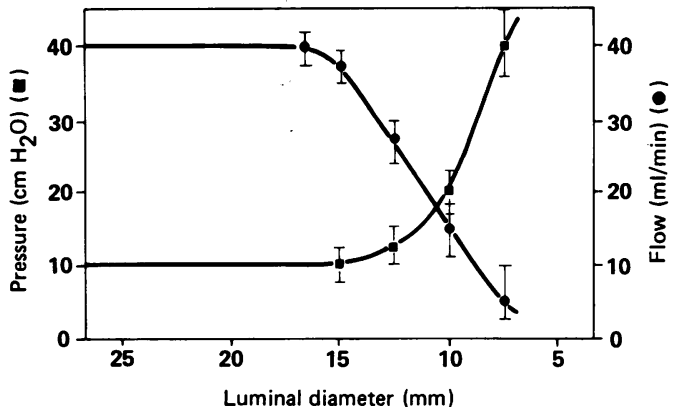

Figure 6: Relation between luminal diameter, flow, and pressure in human small bowel, using a normal saline solution with a $1 \mathrm{~cm}$ long stenosis. $\mathbf{+}=$ mean; $\mathrm{I}=$ standard deviation.

accumulation of the intraluminal content, inducing distension of the loop with a raised intraluminal pressure. Under normal conditions, muscular activity is of major importance in determining intraluminal pressure. When the bowel is stretched and becomes less mobile, the function coefficient of the mucosa probably becomes the decisive factor. ${ }^{2}$ Our experiment does not record the first acute reaction of the gut, but takes into account the material properties of the intestinal wall responsible for the intraluminal pressure of the second phase. The rise in the intraluminal pressure proximal to the stenosis must be progressive, however, as the volume induced distension is time-dependent. ${ }^{4}$ Moreover, if at the onset of obstruction only the segment immediately proximal to the stenosis is considered, the early and late phases extend progressively proximally in the gut up to the stomach; so that for two to five days there will always be a segment of gut in early phase activity pushing the luminal contents into a distal loop which is in late phase activity. ${ }^{3}$ This is limited by our pump system, except for the constant flow induced by our experimental model. The increased motor activity proximal to the obstruction seems to be mediated by atropine-sensitive pathways. ${ }^{5}$ Descending excitatory contractions, proximal to the bolus, are probably mediated through cholinergic synapses. ${ }^{6}$

From these observations and studies we assume that our animal and human model resemble the physiological mechanisms of bowel obstruction.

Clinical observations confirm ${ }^{7}$ that minor stenoses of the gut can be asymptomatic until a critical point is reached at which symptoms begin and proximal accumulation of intestinal content occurs. We had suspected empirically that this critical diameter for symptoms was about $20 \mathrm{~mm}$ or less.

Our experiments confirm that mild to moderate stenoses do not interfere with proximal intraluminal pressure or speed of the transit. From $60 \%$ of the normal diameter and less, proximal pressure rises, with a simultaneous dilatation of the loop and a decreased flow in the distal animal gut. Using human small bowel loops, the critical diameter was found to be at $15 \mathrm{~mm}(60 \%$ of the normal luminal diameter of $25 \mathrm{~mm})$. This diameter was calculated with perfusion of normal saline. In vivo, the presence of undigested particles will increase the viscosity and so lower the critical point at which pressure
Figure 5: Effects of variation of the length of the stenosis on pressure and flow. Saline perfusion of rabbit jejunum at luminal diameters of 4,8 , and $12 \mathrm{~mm}$. 
rises sharply. In operating on patients with Crohn's disease we have noticed ${ }^{8}$ that small bowel stenoses with a diameter larger than $20 \mathrm{~mm}$ are usually asymptomatic, whereas near complete clinical obstruction occurs with stenoses of $15 \mathrm{~mm}$ or less.

The length of the stenoses also modifies the critical diameter, which becomes larger with a longer stricture. We might therefore expect that in Crohn's disease, for example, even moderately tight ( 20 to $25 \mathrm{~mm}$ diameter) but long (100 to 200 $\mathrm{mm}$ ) narrowing of a terminal ileal loop would be associated with occlusive symptoms. On the other hand, several stenoses along the gut do not seem to alter the critical diameter, which remains that of the narrowest stricture. Therefore, in Crohn's disease we recommend a search for undetected stenoses during a laparotomy performed for a single identified stricture. For this purpose, our previously described balloon technique $^{9}$ seems to be useful. Depending on its aetiology, site, diameter, and length, a stricture detected in this manner can be treated by strictureplasty or resection. ${ }^{9}$

In our animal model we experienced bowel rupture at the level of 30 to $35 \%$ of normal size. This is an early mechanical rupture of the small bowel, similar to that occurring in vivo. Well documented studies ${ }^{10-12}$ have emphasised that in the early phase obstructed gut requires a higher blood flow (increasing up to $85 \%$ ). Ischaemic rupture of the bowel wall can therefore be accredited only to the long term effects of an obstruction.

Our experiments confirm the existence of a critical luminal diameter in the rabbit and the human gut. Below this diameter, the normal physiological parameters of the bowel are rapidly changed up to the point of obstruction. The results obtained with this experimental model are consistent with clinical observations ${ }^{13-15}$ and support the need for further pathophysiological studies of fibrosis induced bowel stenosis.
Because it is not possible to extrapolate from experimental conditions to those in a conscious animal or a diseased human, no dogmatic conclusions can be drawn. These studies, however, give support to our empirically derived rules for the management of patients with Crohn's disease suffering from obstructive symptoms. In these patients we consider that any stenosis of less than $15 \mathrm{~mm}$ in diameter ${ }^{8}$ is an indication for operative intervention or dilatation and that stenoses between 15 and $20 \mathrm{~mm}$ provide a relative indication.

1 Summers RW, Yanda R, Prihoda M, Flatt A. Acute intestinal obstruction: an electromyographic study in dogs. Gastro enterology 1983; 85: 1301-6.

2 Enochsson L, Inacio J, Nylander G, Strömberg L. Mechanical compliance of the small intestine related to long-standing obstruction. Acta Chir Scand 1986; 152: 617-21

3 Fleisher DR. On the measurement of intestinal tonus. Gastroenterology 1970; 58: 685-9.

4 Coehlo JCU, Gouma DJ, Moody FG, Li YF, Senninger N. Gastrointestinal motility following small bowel obstruction in the opossum. F Surg Res 1986; 41: 274-8.

5 Prihoda M, Flatt A, Summers RW. Mechanisms of motility changes during acute intestinal obstruction in the dog. $A m$ Physiol 1984; 247: G37-42.

6 Hirst GD. Mechanisms of peristalsis. Br Med Bull 1979; 35 263-8.

7 Alexander-Williams J, Allan A, Morel Ph, Hawker PC, Dykes PW, O'Connor H. The therapeutic dilatation of enteric strictures due to Crohn's disease. Ann R Coll Surg Engl 1986; 68: 95-7.

8 Alexander-Williams J, Haynes IG. Conservative operations for Crohn's disease of the small bowel. World $\mathcal{F}$ Surg 1985; 9 945-51.

9 Alexander-Williams J. The technique of intestinal strictureplasty. Int $\mathcal{f}$ Colorect Dis 1986; 1: 54-7.

10 Papanicolaou G, Nikas D, Ahn Y, Condos S, Fielding LP. Regional blood flow and water content of the obstructed small intestine. Arch Surg 1985; 120:926-32.

11 Ruf W, Suehiro G, Suehiro A. Small intestinal blood flow after 48 hours ileus, prostigmin and manual decompression. $Z$ Exp Z Chirug 1980; 13: 267-73.

12 Eade MN, Ginn RW. The distribution of blood flow along the small intestine of the dog. Proc Soc Exp Biol Med 1978; 157 390-2.

13 Friedman G, Wolfe BS, Waye JD. Correlation of cineradiographic and intra-luminal pressure changes in the human duodenum: an analysis of the functional significance of monophasic waves. Gastroenterology $1965 ; 43$ : 37-49.

14 Schuffler MD, Rohrmann CA, Templeton FE. The radiologic manifestations of idiopathic intestinal pseudo-obstruction.

Am $\mathcal{F}$ Roentgenol Radium Ther Nucl Med 1976; 127: 729-36.
15 Summers RW, Anuras S, Green J. Jejunal manometry patterns in health, partial intestinal obstruction, and pseudo-obstruction. Gastroenterology 1983; 85: 1290-300. 